\title{
Pharmaceutical Cocrystals: Formulation Approaches to Develop Robust Drug Products
}

Naga Kiran Duggirala, ${ }^{a, c}$, Shawn M. LaCasse ${ }^{b}$, Michael J. Zaworotko ${ }^{c}$, Joseph F. Krzyzaniak ${ }^{b}$ and Kapildev K. Arora ${ }^{b^{*}}$

${ }^{a}$ Current address: Drug Product Technologies, Amgen Inc., One Amgen Center Drive, Thousand Oaks, California 91320, USA

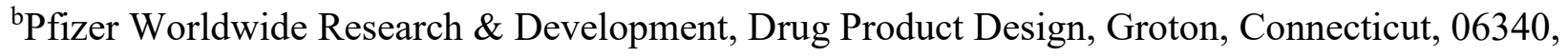
USA

${ }^{\mathrm{c}}$ Department of Chemical Sciences and Bernal Institute, University of Limerick, Ireland 
ERTLPG: Physicochemical stability data for Fitz and Jet milled lots

ERTLPG is physically and chemically stable up to $\sim 20$ months when Fitz milled and stored at 25 and $40{ }^{\circ} \mathrm{C}$ at 75 and $93 \% \mathrm{RH}$. Jet milled ERTLPG material was analyzed up to 18 months and no physical or chemical stability concerns were determined (see table 1 and 2).

Table S1. Fitz and Jet milled cocrystal samples stored at different temperatures with varying relative humidity followed by analyses utilizing semi-quant Raman method

\begin{tabular}{|c|c|c|c|c|c|c|c|c|}
\hline \multirow[t]{3}{*}{ ERTLPG } & \multicolumn{2}{|c|}{ Storage conditions } & \multicolumn{6}{|c|}{ Storage time in months } \\
\hline & & & 2.3 & 2.8 & 2.9 & 5.0 & 17.5 & 19.7 \\
\hline & $\mathbf{T}\left({ }^{\circ} \mathbf{C}\right)$ & RH (\%) & & & & & & \\
\hline \multirow{4}{*}{ Fitz milled } & & 75 & & \multirow{4}{*}{ NT } & & & \multirow{4}{*}{ NT } & \\
\hline & $2 J$ & 93 & & & & & & \\
\hline & \multirow{2}{*}{40} & 75 & & & & & & \\
\hline & & 93 & & & & & & \\
\hline & & & & & & & & \\
\hline \multirow{4}{*}{ Jet milled } & \multirow{2}{*}{25} & 75 & NT & & NT & NT & & NT \\
\hline & & 93 & & & & & & \\
\hline & \multirow{2}{*}{40} & 75 & & & & & & \\
\hline & & 93 & & & & & & \\
\hline
\end{tabular}

$* \mathrm{NT}-$ not tested

- no amorphous content was observed by Raman method

Table S2. Fitz and Jet milled cocrystal samples stored at different temperatures with varying relative humidity followed by analyses HPLC for any chemical degradation

\begin{tabular}{|l|c|c|}
\hline \multicolumn{1}{|c|}{ Sample Name } & PF-04971729 Assay & Total impurities \\
& (\%) & (\%) \\
\hline ERTLPG (Fitz milled, $\left.25^{\circ} \mathrm{C} / 75 \% \mathrm{RH}\right)$ & 74.48 & NMT 0.05 \\
\hline ERTLPG (Fitz milled, $\left.40^{\circ} \mathrm{C} / 75 \% \mathrm{RH}\right)$ & 74.04 & NMT 0.05 \\
\hline ERTLPG (Fitz milled, $\left.25^{\circ} \mathrm{C} / 93 \% \mathrm{RH}\right)$ & 75.13 & NMT 0.05 \\
\hline ERTLPG (Fitz milled, $\left.40^{\circ} \mathrm{C} / 93 \% \mathrm{RH}\right)$ & 74.06 & NMT 0.05 \\
\hline ERTLPG (Jet milled, $\left.25^{\circ} \mathrm{C} / 75 \% \mathrm{RH}\right)$ & 77.85 & NMT 0.05 \\
\hline ERTLPG (Jet milled, $\left.40^{\circ} \mathrm{C} / 75 \% \mathrm{RH}\right)$ & 77.51 & NMT 0.05 \\
\hline
\end{tabular}




\begin{tabular}{|l|c|c|}
\hline ERTLPG (Jet milled, $\left.25^{\circ} \mathrm{C} / 93 \% \mathrm{RH}\right)$ & 77.23 & NMT 0.05 \\
\hline ERTLPG (Jet milled, $\left.40^{\circ} \mathrm{C} / 93 \% \mathrm{RH}\right)$ & 77.05 & NMT 0.05 \\
\hline
\end{tabular}

The Fitz milled samples were stored for $\sim 19.7$ month Jet milled samples for $\sim 17.5$ months.

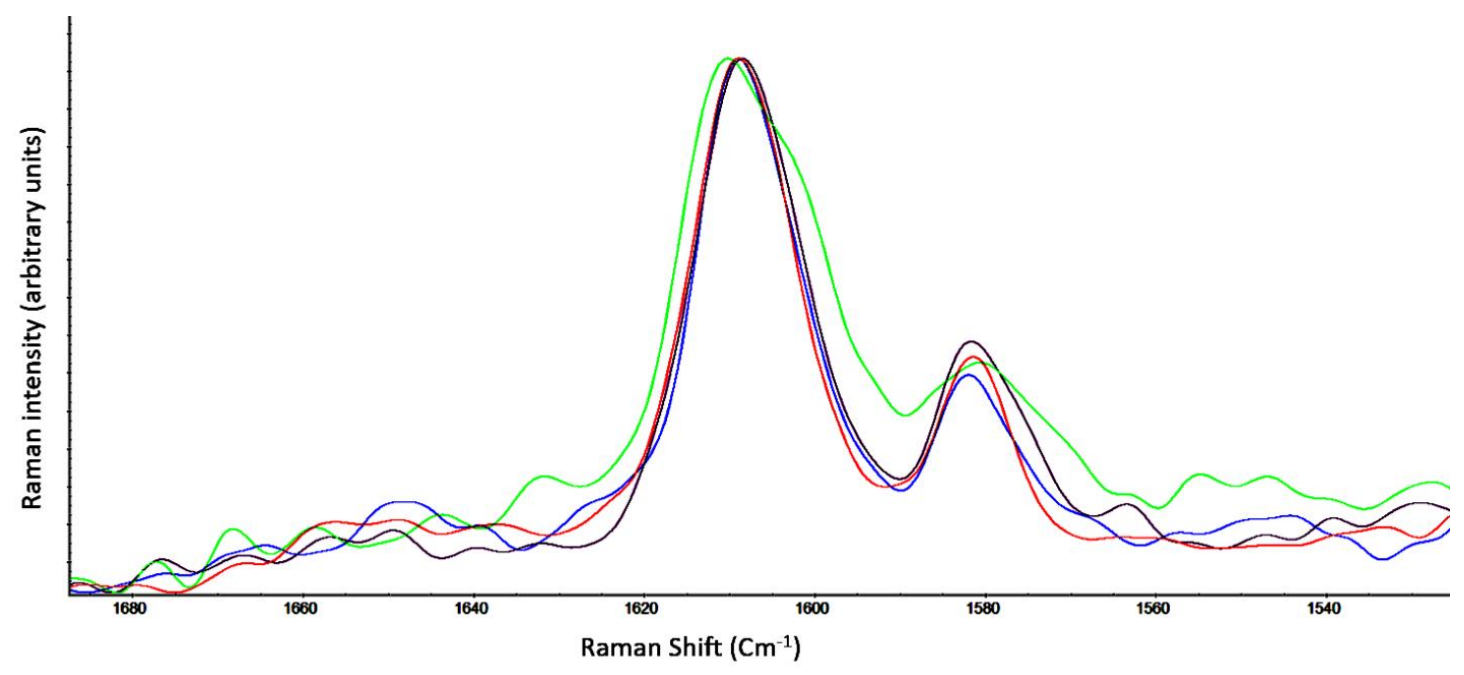

Figure S1. Overlay of ERTLPG cocrystal tablet (red) and 100\% amorphous ertugliflozin tablet (green) with two stability conditions - blue $\left(25^{\circ} \mathrm{C} / 60 \% \mathrm{RH} 10\right.$ months $)$, which shows essentially no change and purple $\left(30{ }^{\circ} \mathrm{C} / 75 \% \mathrm{RH} 10\right.$ months) which shows signs of peak broadening, indicating formation of amorphous ertugliflozin, i.e., dissociation of the ERTLPG cocrystal

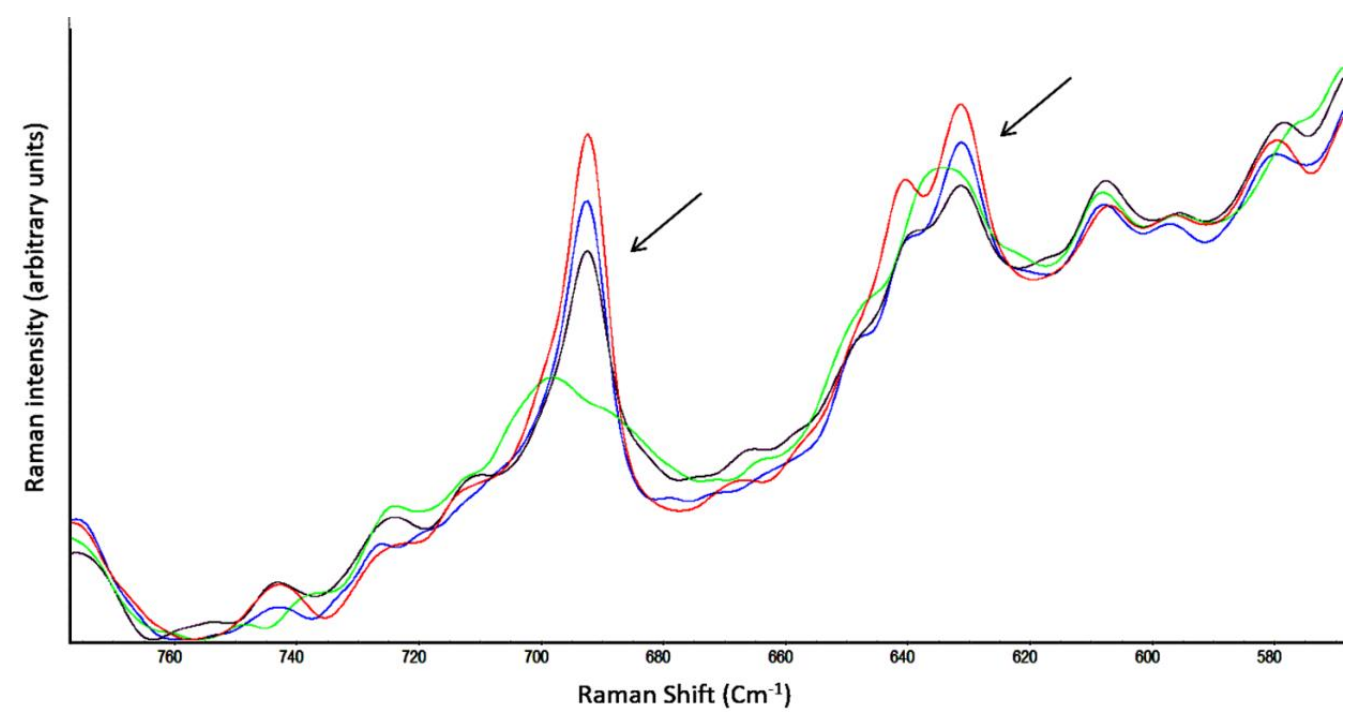


Figure S2. Overlay of ERTLPG cocrystal tablet (red) and 100\% amorphous ertugliflozin tablet (green) with two stability conditions - blue $\left(25^{\circ} \mathrm{C} / 60 \% \mathrm{RH} 10\right.$ months), which shows essentially no change and purple $\left(30^{\circ} \mathrm{C} / 75 \% \mathrm{RH} 10\right.$ months) which shows signs of peak broadening and loss of peak intensity, formation of amorphous ertugliflozin, i.e., dissociation of the ERTLPG cocrystal.

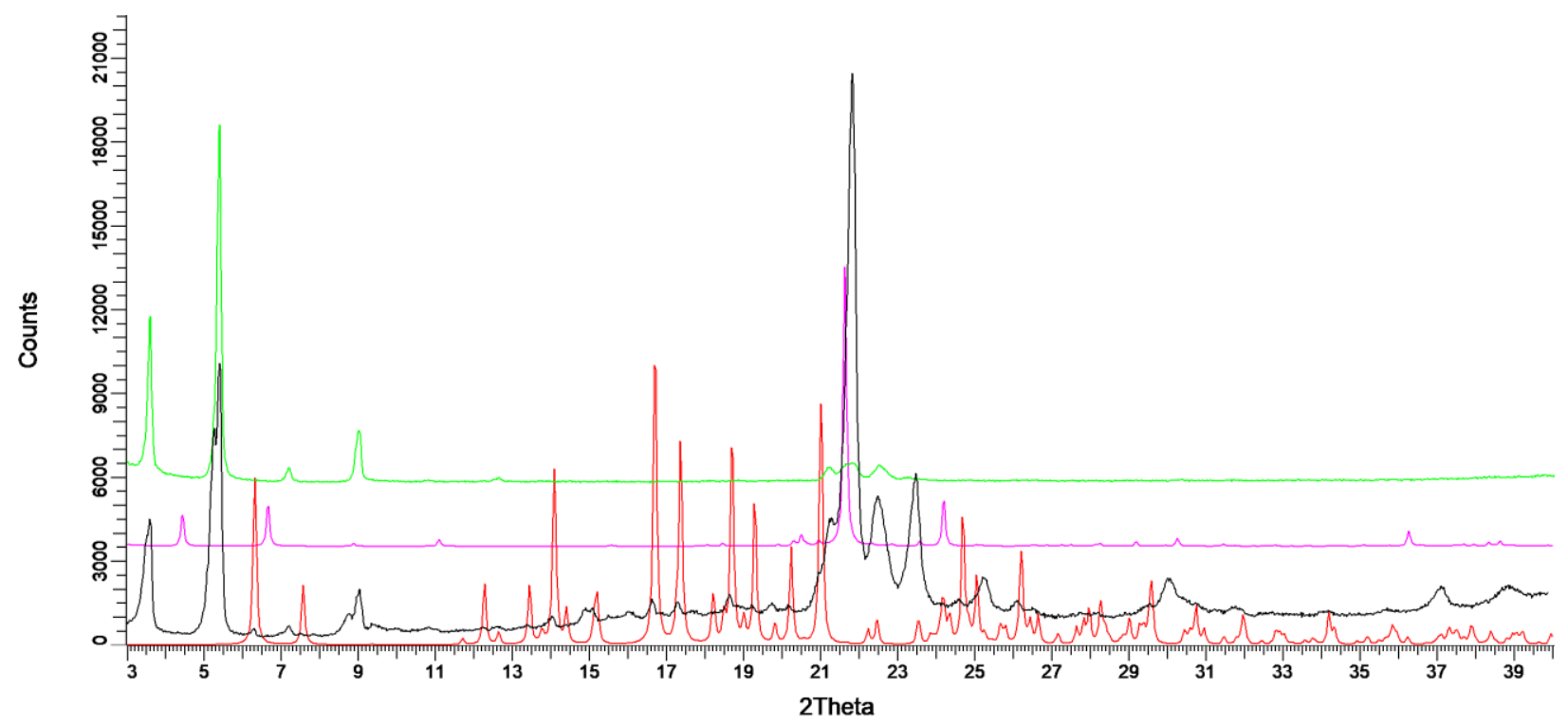

Figure S3. PXRD Overlay of ERTLPG cocrystal; red-calculated PXRD pattern of ERTLPG, black-binary mixture of ERTLPG $+\mathrm{MgSt}$ (4-week data point at $40{ }^{\circ} \mathrm{C} / 75 \% \mathrm{RH}$ ), pink-calculated stearic acid PXRD, and green-MgSt. Peak at $21.6^{\circ}$ 2theta is stearic acid diffraction peak observed in stressed tablet (black).

Eutectic Point measurement of ERTLPG cocrystal

At the eutectic

\begin{tabular}{|l|l|l|l|l|l|}
\hline ERT (M) & LPG (M) & $\mathrm{pH}$ & $\begin{array}{l}\text { Solid Form } \\
(\text { PXRD) }\end{array}$ & $\begin{array}{l}\text { Solubility of } \\
\text { ERTLPG (M) }\end{array}$ & Ksp \\
\hline 0.003433 & 1.746437 & 1.72 & ERTLPG + LPG & 0.077436 & 0.005849 \\
\hline
\end{tabular}

